\section{Glenzocimab does not impact glycoprotein VI-dependent inflammatory hemostasis}

Glycoprotein VI (GPVI), the main platelet receptor for collagen, has emerged as a new target for antithrombotic therapy because its genetic deficiency or pharmacological blocking inhibits platelet aggregation and experimental thrombosis without increasing bleeding time. ${ }^{1-5}$ While these data have stimulated the development of new antiplatelet drugs targeting GPVI, recent findings have indicated that GPVI is essential for repair of neutrophilinduced vascular injury in various inflamed organs and tissues. $^{6-9}$ It thus appears important to assess and anticipate the yet uninvestigated risk of inflammation-induced bleeding under GPVI antagonists, especially considering that inflammation is a component of various thrombotic diseases. In that respect, it is worth noting that neutrophil mobilization is a predictor of hemorrhagic transformation of ischemic stroke ${ }^{10}$ and contributes to intraplaque hemorrhage ${ }^{11}$ which is known to precipitate plaque rupture and the clinical expression of atherosclerosis.

Among the newly developed drugs targeting GPVI, ACT017 (Glenzocimab, Acticor Biotech) is a humanized antibody fragment $(\mathrm{Fab})$ that has already completed its phase I clinical trial in healthy volunteers ${ }^{12}$ and has just entered a phase II trial in stroke patients (Acute Ischemic Stroke Interventional Study "ACTIMIS", clinicaltrials gov. Identifier: NCT03803007). ACT017 binds to human GPVI and inhibits the procoagulant activity and aggregation of collagen-stimulated platelets, as well as platelet adhesion and thrombus formation onto collagen surfaces under arterial flow conditions. ${ }^{1,13,14}$ The inhibitory action of ACT017 occurs without causing thrombocytopenia or depletion of GPVI, and is not associated with spontaneous bleeding events or increased bleeding time. ${ }^{14}$ Nevertheless, whereas preclinical bleeding time tests can help evaluate the risk of bleeding associated with trauma or surgery, they may not predict the risk of bleeding associated with inflammation. ${ }^{15}$ Here, using the cutaneous reverse passive Arthus reaction ( $\mathrm{rpA}$ ) as a model situation where GPVI plays a major role in inflammatory hemostasis, we investigated whether ACT017 increases the risk of inflammation-induced bleeding.

We first assessed the contribution of GPVI to the prevention of inflammation-induced bleeding by platelets in the brain and lungs. In agreement with previous results obtained with an antibody causing depletion of mouse Gpvi, ${ }^{16,17}$ there was no cerebral hemorrhage in any of the $\mathrm{Gpvi}^{-1-}$ mice subjected to transient (90 minutes) middle cerebral artery occlusion (Figure 1A). In contrast, cerebral hemorrhage occurred in all mice that had been rendered severely thrombocytopenic by the mean of a plateletdepleting antibody (Figure 1A). Genetic deficiency in $G P V I$ was not associated with an increased bleeding risk in the model of acute lung injury induced by inhalation of Pseudomonas aeroginosa endotoxin either (Figure 1B). In the cutaneous $\mathrm{rpA}$, as predicted by previous reports, ${ }^{6,7,9}$ $G P V I^{-1-}$ mice developed skin bleeding at the inflammatory reaction site, a bleeding phenotype that was seen neither in $G P V I^{+/+}$mice nor in GPVI ${ }^{+/-}$mice, which expressed half of normal GPVI surface levels (Figure 1C and D). Taken together, these results are consistent with evidence that GPVI is dispensable for hemostasis in the inflamed brain and lungs ${ }^{6,16-18}$ but primarily involved in the prevention of bleeding in the rpA-inflamed skin. Notably, they further indicate that $50 \%$ of normal GPVI surface levels are sufficient for hemostasis during the cutaneous $\mathrm{rpA}$.
The ability of ACT017 to inhibit collagen/GPVI interactions and their functional consequences has been previously demonstrated in humans and in nonhuman primates. ${ }^{14}$ However, it has not been tested in $h G P V I$ mice. We thus verified the activity of ACT017 against GPVI from $h G P V I$ mice. Like its murine precursor Fab 90121 , ACT017 added to whole blood from $h$ GPVI mice caused a drastic reduction in platelet adhesion and aggregation onto collagen fibers under arterial and venous flow conditions (Figure 2A and B; Online Supplementary Movie). We next tested whether $h G P V I$ mice treated with therapeutic $(16 \mathrm{mg} / \mathrm{kg}$ ) or higher doses of ACT017 (32 and $64 \mathrm{mg} / \mathrm{kg}$ ) were sensitized to inflammation-induced bleeding during the cutaneous rpA. No bleeding occurred in ACT017treated $h$ GPVI mice, whatever the dose of ACT017 used (data not shown, Figure 2C and D). There was no bleeding either when ACT017 at the highest dose tested (64 $\mathrm{mg} / \mathrm{kg}$ ) was given through a continuous infusion over the 4 hours of rpA (data not shown). The absence of bleeding in $h G P V I$ mice treated with ACT017 was in contrast to the petechial bleeding observed in $G P V I^{-/}$mice (Figure 1) and in platelet-depleted $G P V I^{+/+}$and $h G P V I$ mice (Figures $1 \mathrm{C}$ and $\mathrm{D}$, and $2 \mathrm{C}$ and $\mathrm{D}$ ), which is known to be a consequence of neutrophil recruitment. ${ }^{7}$ Absence of bleeding in rpA-challenged $h G P V I$ mice treated with ACT017 was not due to altered neutrophil recruitment as this was comparable to that in $h G P V I$ mice (Figure 2E). Interestingly, the latter result indicates that ACT017 does not impact neutrophil recruitment, at least in this model. Importantly, ACT017 did not alter platelet recruitment to the inflamed skin either (Figure 2F). The latter result underscores a major difference between the impact of genetic deficiency in GPVI and that of GPVI blocking by ACT017. In fact, bleeding in rpA-challenged $G p v i^{-1 /}$ mice was previously shown to be associated with reduced platelet recruitment at the reaction site.

Solid tumors represent another inflammatory situation in which platelets were shown to continuously prevent leukocyte-induced bleeding and recent data have suggested that GPVI could be central to this function. ${ }^{19}$ Like in the cutaneous $\mathrm{rpA}$, acute treatment of $h G P V I$ mice bearing skin tumors with ACT017 did not cause tumor bleeding. The absence of effect of ACT017 on tumor vessel stability was in contrast to the effect of acute depletion of platelets, which caused tumor bleeding (Figure 2F).

In conclusion, in addition to confirming that GPVI is not required for inflammation-associated hemostasis in the brain and lungs, our results show that pharmacological blockade of GPVI by ACT017 does not impair GPVIdependent inflammatory hemostasis. There are several non-exclusive reasons that could explain why pharmacological inhibition of GPVI by ACT017 does not impair the vasculoprotective recruitment of platelets during the cutaneous rpA. First, it was shown previously that GPVI can co-operate with other platelet receptors like integrin $\alpha 2 \beta 1$ to provide residual collagen-dependent platelet activation when its collagen binding site is blocked pharmacologically. ${ }^{20}$ Furthermore, while ACT017 blocks the interactions between GPVI and collagen, it remains unknown whether ACT017 has similar blocking effects towards the other ligands of GPVI. Besides collagen, fibrin(ogen) and a number of adhesive proteins of the vessel wall have been reported as GPVI ligands (e.g., laminin, fibronectin and vitronectin) and could thus provide redundant binding mechanisms. Moreover, were collagen to be one of the ligands supporting the adhesion of platelets to inflamed skin vessels, it is interesting to note that despite a drastic reduction in platelet adhesion and 
A

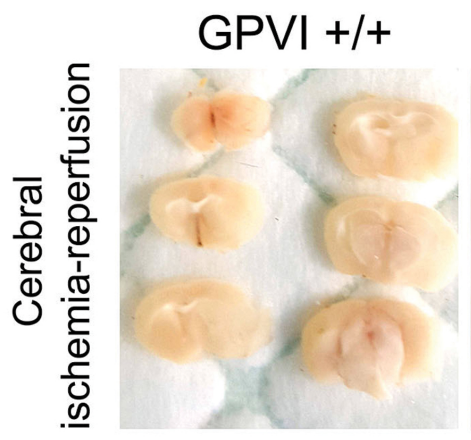

B

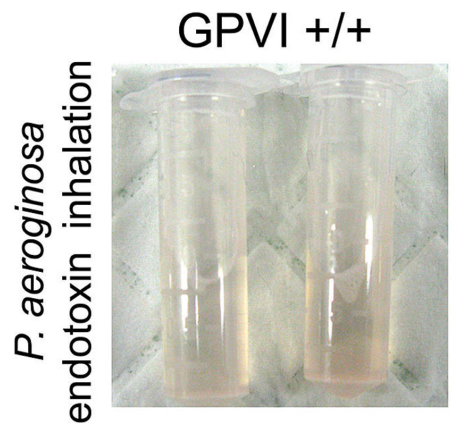

GPVI -/-

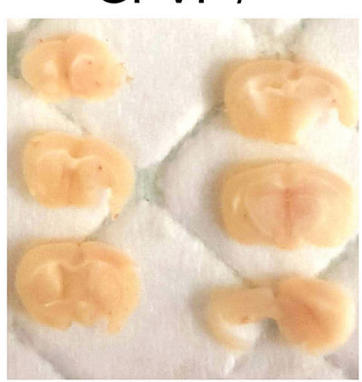

GPVI -/-

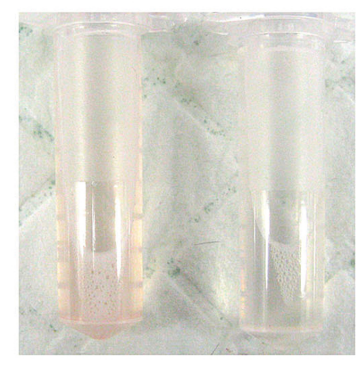

Platelet-depleted

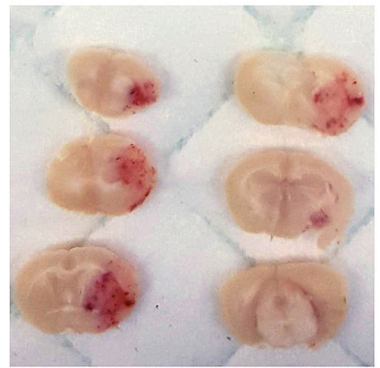

Platelet-depleted

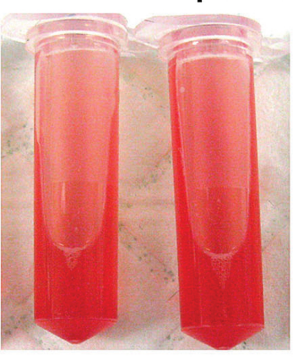

C
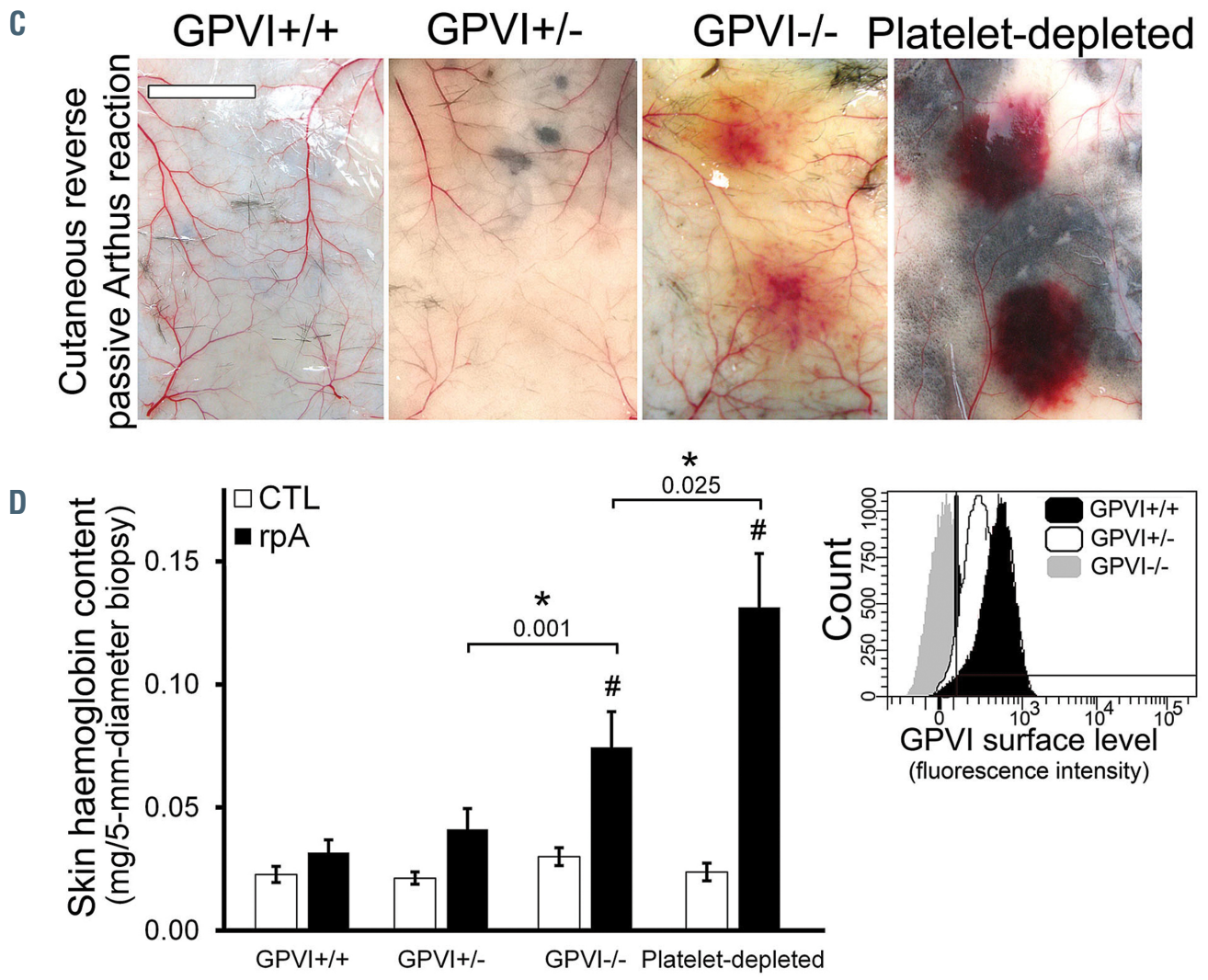

Figure 1. Contribution of glycoprotein VI to inflammation-associated hemostasis. The contribution of glycoprotein VI (GPVI) to inflammation-associated hemostasis was determined in three different models of acute inflammation. (A) Representative images of brain sections taken 24 hours after $\mathrm{GPVI}^{+/+}$, GPVI ${ }^{-}$ $\mathrm{GPVI}^{-}$, and platelet-depleted mice were subjected to 90 minutes transient middle cerebral artery occlusion (tMCAO). Note that tMCAO caused bleeding only in platelet-depleted mice. The images are representative of $n=6$ mice per group. (B) Representative images of the bronchoalveolar lavage fluid from Gpvi ${ }^{+/}$, Gpvi , , and platelet-depleted mice collected 24 hours after lipopolysaccharide inhalation. The images are representative of $n=8$ mice per group. (C and $D)$ Effect of partial or complete GPVI deficiency on inflammatory bleeding during the cutaneous reverse passive Arthus reaction (rpA). (C) Representative images of the skin of $\mathrm{GPVI}^{+/+}, \mathrm{GPVI}{ }^{\circ}, \mathrm{GPVI}$, and platelet-depleted mice after 4 hours of rpA. The images are representative of $\mathrm{n}=7-10 \mathrm{mice}$ per group. Bar $=500 \mathrm{um}$. (D) Skin hemoglobin content after 4 hours of rpA. \# indicates a significant difference $(P<0.05)$ from the $\mathrm{rpA} \mathrm{GPVI}^{+/+}$group, $\mathrm{n}=14-20 \mathrm{skin}$ biopsies per group. Inset: Representative histogram of flow cytometry analysis of GPVI surface levels in $\mathrm{GPVI}^{+/+}, \mathrm{GPVI}^{+/}$, and $\mathrm{GPVI}^{\%}$ mice, as assessed using the $\mathrm{JAQ} 1$ antibody to representative mouse GPVI. 


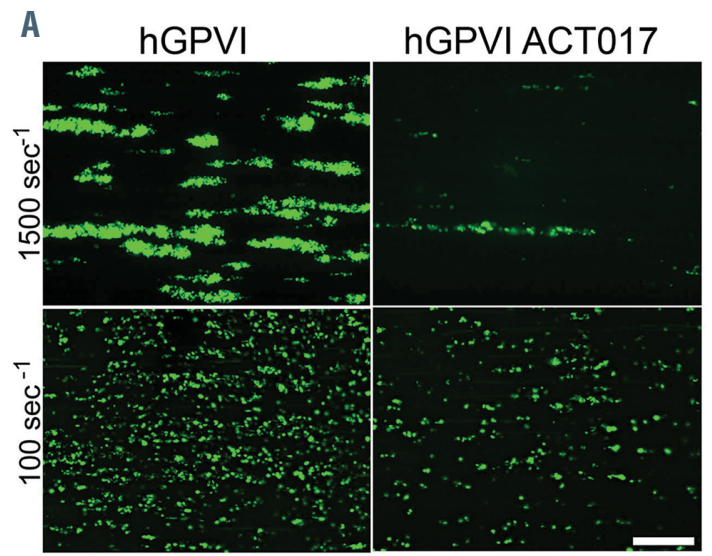

B
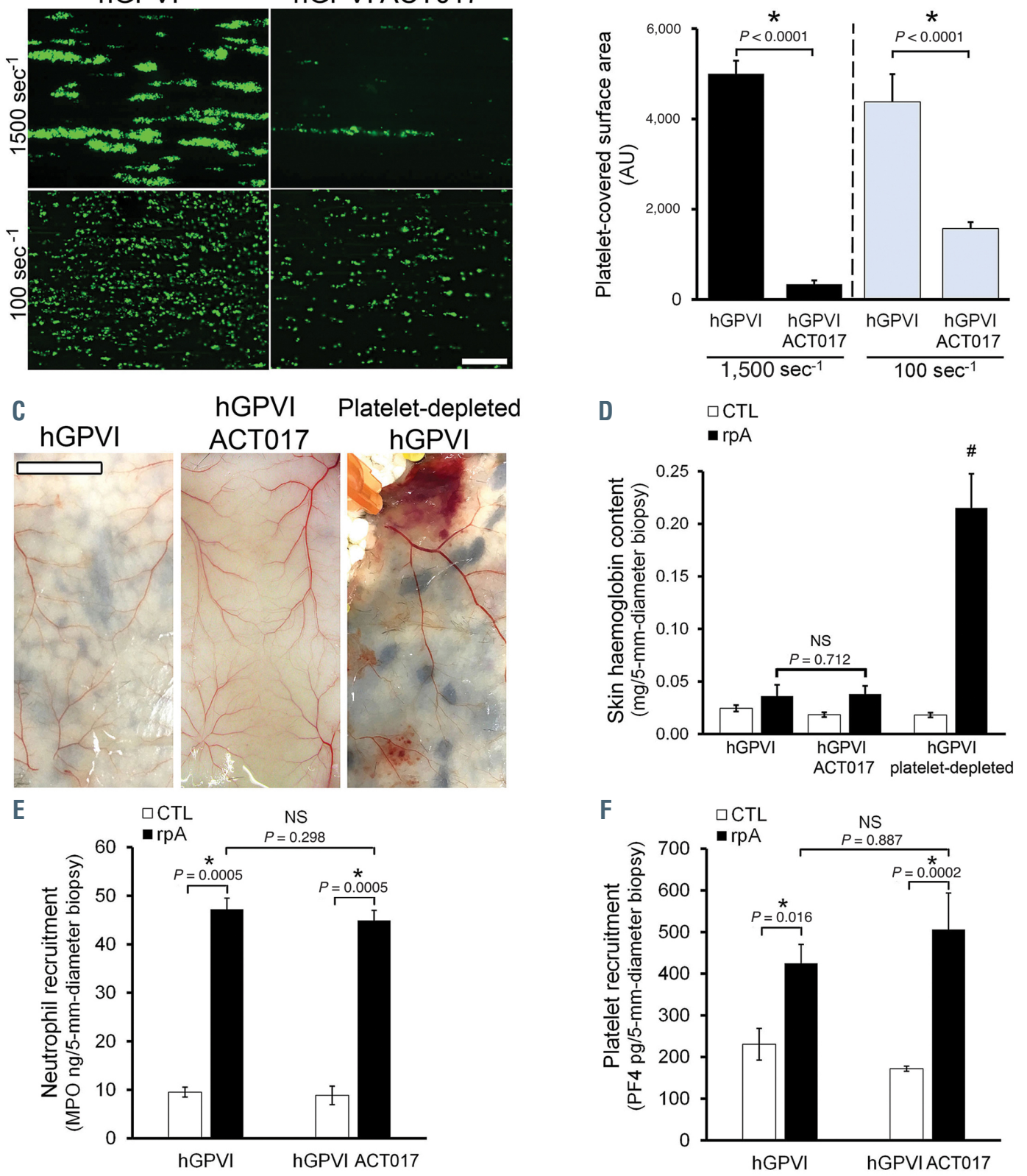

Figure 2. Impact of ACT017 on glycoprotein VI-dependent hemostasis. (A and B) Citrated whole blood from hGPVI mice was labeled with the fluorochrome DiOC6, incubated or not with ACT017 $(80 \mu \mathrm{g} / \mathrm{mL})$ for 10 minutes, and perfused at a wall shear rate of $1,500 \mathrm{~s}^{-1}$ or $100 \mathrm{~s}^{-1}$ for 3 minutes over a collagen-coated surface. Bar=50 um. (A) Representative images of platelet coverage at the end of the perfusion. (B) Mean surface areas covered by platelets calculated from 20 different fields taken with a 20x objective along channels from four different runs (five fields per run). (C) Representative images of the skin of $h$ GPVI mice treated or not with ACT017 (64 mg/kg) after 4 hours of reverse passive Arthus reaction (rpA). The images are representative of $n=4-9$ mice per group. Bar $=500 \mu \mathrm{m}$. (D) Skin hemoglobin content after 4 hours of rpA. \# indicates a significant difference $(P<0.05)$ from the rpA $h \mathrm{GPVI}$ group, $\mathrm{n}=6$ - $18 \mathrm{skin}$ biopsies per group. (E and F) Skin myeloperoxidase (E) and platelet factor 4 (PF4) (F) content after 2 hours of rpA, as assessed by enzyme-linked immunosorbent assay; $\mathrm{n}=12$ skin biopsies per group.

aggregation onto fibrillar collagen in the presence of ACT017 (Figure 2A and B; Online Supplementary Movie), residual platelet adhesion was observed at both arterial and venous blood flow. Considering that ACT017 has no effect on platelet recruitment during cutaneous $\mathrm{rpA}$ and that previous results have shown that individual platelets and platelet monolayers ensure hemostasis at sites of mild inflammatory vascular injury, ${ }^{15}$ such residual interactions with collagen could be sufficient for inflammatory hemostasis. Previous studies have also shown that platelets are particularly efficient in maintaining vascular integrity in inflamed organs, as platelet counts as low as $10 \%$ can support this function. ${ }^{15}$ Consistent with this notion, $G p v i^{+/-}$mice with half of normal GPVI surface levels showed normal hemostasis during the cutaneous $\mathrm{rpA}$ (Figure $1 \mathrm{C}$ and D). All in all, our results indicate that the highly favorable safety profile of ACT017 suggested by previous results in bleeding time assays and by the absence of adverse bleeding events in the phase I clinical trial $^{12}$ also applies to inflammatory situations. Whether 
the safety profile of ACT017 still holds true when combining it with other drugs like recombinant tissue-type plasminogen activator remains to ascertain, but the absence of effect of ACT017 on platelet recruitment to the inflamed vasculature suggests there is a realistic chance for it to be maintained.

Soumaya Jadoui, ${ }^{1^{*}}$ Ophélie Le Chapelain, ${ }^{1^{*}}$

Véronique Ollivier, ${ }^{1}$ Ali Mostefa-Kara, ${ }^{1}$ Lucas Di Meglio, ${ }^{1}$

Sébastien Dupont, ${ }^{1}$ Angèle Gros, ${ }^{1}$ Mialitiana Solo

Nomenjanahary, ${ }^{1}$ Jean-Philippe Desilles, ${ }^{1,2}$ Mikaël Mazighi, ${ }^{1,2}$

Bernhard Nieswandt, ${ }^{3}$ Stéphane Loyau, ${ }^{1}$ Martine

Jandrot-Perrus, ${ }^{1}$ Pierre H. Mangin ${ }^{4}$ and Benoit Ho-Tin-Noé ${ }^{1}$

${ }^{1}$ Université de Paris, LVTS, INSERM U1148, Paris, France;

${ }^{2}$ Department of Interventional Neuroradiology, Rothschild

Foundation Hospital, Paris, France; ${ }^{3}$ University Hospital

Würzburg, Rudolf Virchow Center for Experimental Biomedicine,

Würzburg, Germany and ${ }^{4}$ Université de Strasbourg, INSERM,

EFS Grand-Est, BPPS UMR-S1255, FMTS, Strasbourg, France

*SJ and OLC contributed equally as co-first authors.

Correspondence:

BENOITHO-TIN-NOE' - benoit.ho-tin-noe@inserm.fr

doi:10.3324/haematol.2020.270439

Received: August 25, 2020.

Accepted: December 21, 2020.

Pre-published: December 30, 2020.

Disclosures: MJ-P is the founder of Acticor Biotech; all other authors have no conflicts of interest to disclose.

Contributions: SJ, OLC, VO, MSN, AM,LDM, SD, AG, and SL performed research and data analysis; JPD, MM, MJP, BN, and PHM provided critical feedback and helped shape the research; BTHN supervised the study, performed research and data analysis, and wrote the manuscript with support of SJ, OLC, VO, SD and MJP; all authors discussed the results and contributed to the final manuscript.

Funding: this work was supported by grants from INCA (2016-09/435/NI-KA), La Fondation ARC (PJA 20151203107), La Fondation pour la Recherche Médicale (grant \#DPC20171138959), and by public grants overseen by the French National Research Agency (ANR) as part of the Investments for the Future program (PIA) under grant agreement No. ANR-18-RHUS-0001 (RHU Booster) and BPI (CMI2 project TherAVC2.0); SJ was awarded a grant from la Société Française d'Hématologie.

\section{References}

1. Mangin PH, Tang CJ, Bourdon C, et al. A humanized glycoprotein VI (GPVI) mouse model to assess the antithrombotic efficacies of anti-GPVI agents. J Pharmacol Exp Ther. 2012;341(1):156-163.

2. Dütting S, Bender M, Nieswandt B. Platelet GPVI: a target for antithrombotic therapy?! Trends Pharmacol Sci. 2012;33(11):583590

3. Jiang P, Jandrot-Perrus M. New advances in treating thrombotic diseases: GPVI as a platelet drug target. Drug Discov Today. 2014 19(9):1471-1475

4. Stegner D, Haining EJ, Nieswandt B. Targeting glycoprotein vi and the immunoreceptor tyrosine-based activation motif signaling pathway. Arterioscler Thromb Vasc Biol. 2014;34(8):1615-1620.

5. Rayes J, Watson SP, Nieswandt B. Functional significance of the platelet immune receptors GPVI and CLEC-2. J Clin Invest. 2019; 129(1):12-23

6. Rayes J, Jadoui S, Lax S, et al. The contribution of platelet glycoprotein receptors to inflammatory bleeding prevention is stimulus and organ dependent. Haematologica. 2018;103(6):e256-e258.

7. Gros A, Syvannarath V, Lamrani L, et al. Single platelets seal neutrophil-induced vascular breaches via GPVI during immune-complex-mediated inflammation in mice. Blood. 2015;126(8):1017-1026.

8. Hillgruber C, Pöppelmann B, Weishaupt C, et al. Blocking neutrophil diapedesis prevents hemorrhage during thrombocytopenia. J Exp Med. 2015;212(8):1255-1266

9. Boulaftali Y, Hess PR, Getz TM, et al. Platelet ITAM signaling is critical for vascular integrity in infammation. J Clin Invest. 2013 123(2):908-916

10. Maestrini I, Strbian D, Gautier S, et al. Higher neutrophil counts before thrombolysis for cerebral ischemia predict worse outcomes. Neurology. 2015;85(16):1408-1416.

11. Michel JB, Martin-Ventura IL, Nicoletti A, Ho-Tin-Noé B. Pathology of human plaque vulnerability: mechanisms and consequences of intraplaque haemorrhages. Atherosclerosis. 2014;234(2):311-319.

12. Voors-Pette C, Lebozec K, Dogterom P, et al. Safety and tolerability, pharmacokinetics, and pharmacodynamics of ACT017, an antiplatelet GPVI (glycoprotein VI) Fab: first-in-human healthy volunteer trial. Arterioscler Thromb Vasc Biol. 2019;39(5):956-964.

13. Lecut C, Feeney LA, Kingsbury G, et al. Human platelet glycoprotein VI function is antagonized by monoclonal antibody-derived fab fragments. J Thromb Haemost. 2003;1(12):2653-2662.

14. Lebozec K, Jandrot-Perrus M, Avenard G, Favre-Bulle O, Billiald P. Design, development and characterization of ACT017, a humanized Fab that blocks platelet's glycoprotein VI function without causing bleeding risks. MAbs. 2017;9(6):945-958.

15. Ho-Tin-Noé B, Boulaftali Y, Camerer E. Platelets and vascular integrity: how platelets prevent bleeding in inflammation. Blood. 2018;131(3):277-288.

16. Kraft P, Schuhmann MK, Fluri F, et al. Efficacy and safety of platelet glycoprotein receptor blockade in aged and comorbid mice with acute experimental stroke. Stroke. 2015;46(12):3502-3506.

17. Kleinschnitz C, Pozgajova M, Pham M, Bendszus M, Nieswandt B, Stoll G. Targeting platelets in acute experimental stroke: impact of glycoprotein Ib, VI, and IIb/IIIa blockade on infarct size, functional outcome, and intracranial bleeding. Circulation. 2007;115(17):23232330

18. Claushuis TAM, de Vos AF, Nieswandt B, et al. Platelet glycoprotein VI aids in local immunity during pneumonia-derived sepsis caused by gram-negative bacteria. Blood. 2018;131(8):864-876.

19. Volz J, Mammadova-Bach E, Gil-Pulido J, et al. Inhibition of platelet GPVI induces intratumor hemorrhage and increases efficacy of chemotherapy in mice. Blood. 2019;133(25):2696-2706.

20. Nieswandt B, Brakebusch C, Bergmeier W, et al. Glycoprotein VI but not $\alpha 2 \beta 1$ integrin is essential for platelet interaction with collagen. EMBO J. 2001;20(9):2120-2130. 